\title{
Carotid Atherosclerosis in Ischemic Cerebrovascular Patients
}

\author{
Ai Juan Zhanga, c, Ai Yuan Zhang ${ }^{\mathrm{b}}$, Chi Zhong ${ }^{\mathrm{a}}$
}

\begin{abstract}
Background: Cerebral emboli resulting from atherosclerosis at the carotid bifurcation is a major cause of ischemic stroke. A convenient and prompt evaluation is necessary for secondary prevention and treatment.
\end{abstract}

Methods: In this study, one hundred and thirty eight patients with cerebral ischemic events were enrolled; 100 patients with nonischemic cerebral diseases were enrolled as controls. Noninvasive ultrasound was used to measure the atherosclerotic plaques and intima-media thickness (IMT) of carotid and femoral artery.

Results: Our results showed that patients in study group had higher incidence and severity of carotid and femoral plaques, and higher mean intima-media thickness (IMT) at both the carotid and femoral sites compared with that of controls $(p<0.01)$. Carotid atherosclerosis were highly prone to have instability plaques in study group $(\mathrm{p}$ $<0.001)$.

Conclusions: This cross-sectional study showed that, the prevalence of carotid atherosclerosis and the unstable plaques were higher in cerebral ischemic patients.

Keywords: Carotid artery; Atherosclerosis; Intima-media thickness; Cerebral ischemic stroke

\section{Introduction}

Extracranial internal carotid artery stenosis (EICAS) accounts for approximately $25 \%$ of ischemic stroke. Previous

Manuscript accepted for publication March 12, 2009

aDepartment of Neurology, Weifang People's Hospital affiliated to Weifang Medical Colllege, Shandong, China, 261041

bepartment of cardiology, Weifang People's Hospital affiliated to Weifang Medical Colllege, Shandong, China, 261041

${ }^{\mathrm{c} C}$ Corresponding author: Ai Juan Zhang. Email: aijuanzhang@yahoo.com.cn

doi:10.4021/jocmr2009.03.1226 literature has shown that even patients with asymptomatic EICAS require treatment. The soft and mixed plaques are unstable and may lead to rupture or atheroma, their presence might be at least equal to $50 \%$ stenosis [1]. Unstable plaques put patients at high risk for acute ischemic events. Intimamedia thickness (IMT) is positively correlated with atherosclerotic factors, the severity of arterial stenosis, and the resistance of the carotid artery [2]. In this study, we aimed to investigate the EICAS in the cerebral ischemic stroke in a Chinese population.

\section{Materials and Methods}

\section{Patients}

From April 2006 to December 2007, 138 patients (95 male, 43 female, mean age $63.76 \pm 10.79$ years) admitted for cerebral ischemic diseases as transient ischemic attack (TIA) or cerebral infarction were enrolled in this study, all patients were diagnosed according to the NINDS criteria (the National Institute of Neurological Disorder and Stroke). Patients were excluded from the study if they had a cardiac valvular or rhythm disorder which was likely to be associated with cardiogenic embolism.

Another 100 patients with age-, sex-matched (61 male, 39 female, mean age $61.41 \pm 11.53$ years), were enrolled for controls, they were diagnosed as major depression, headache of tension type, Meniere's disease, and encephalitis.

\section{Imaging examination}

All patients were performed brain CT scan and/or MRI at admission. Personal data such as medical history, family history, body mass index (BMI) were recorded. At the day of test, fasting blood were collected for determination of glucose, lipids profile, renal function (Beckman CX-9 Automatic Biochemical Analyzer).

If patients' condition permitted, the high-resolution ultrasound was used to measure common carotid, internal carotid, and femoral artery atherosclerotic plaques. The stability of plaque was determined according to the resound features and defined as soft, mixed and hard plaque. The IMT at $1-1.5 \mathrm{~cm}$ of right common carotid and right femo- 
Table 1. Baseline clinical features of the patients

\begin{tabular}{|c|c|c|c|}
\hline & Patients & Controls & $P$ value \\
\hline Number & 138 & 100 & \\
\hline Age & $63.76 \pm 10.79$ & $61.41 \pm 11.53$ & $>0.05$ \\
\hline BMI & $25.28 \pm 4.61$ & $25.39 \pm 2.81$ & $>0.05$ \\
\hline $\mathrm{M} / \mathrm{F}$ & $95 / 43$ & $61 / 39$ & $>0.05$ \\
\hline Hypertension & 99 (71.74\%) & $55(55 \%)$ & $<0.01$ \\
\hline Systolic pressure & $150.85 \pm 24.64$ & $140.27 \pm 22.34$ & $<0.01$ \\
\hline Diastolic pressure & $87.46 \pm 14.29$ & $83.47 \pm 12.07$ & $<0.05$ \\
\hline $\mathrm{T}-\mathrm{Ch}$ & $4.85 \pm 1.13$ & $4.99 \pm 1.0$ & $>0.05$ \\
\hline TG & $1.85 \pm 2.16$ & $1.65 \pm 1.35$ & $>0.05$ \\
\hline HDL & $1.13 \pm 0.34$ & $1.26 \pm 0.37$ & $<0.05$ \\
\hline LDL & $2.93 \pm 0.89$ & $3.07 \pm 0.90$ & $>0.05$ \\
\hline Urea & $5.50 \pm 2.05$ & $4.71 \pm 1.14$ & $<0.01$ \\
\hline Creatinine & $99.61 \pm 22.15$ & $88.13 \pm 19.27$ & $<0.001$ \\
\hline Diabetes history & $33(23.91 \%)$ & $15(15 \%)$ & $>0.05$ \\
\hline Glucose & $5.78 \pm 2.08$ & $5.07 \pm 1.74$ & $<0.05$ \\
\hline
\end{tabular}

IMT: intima-media thickness; BMI: body mass index; M: male; F: female; T-Ch: total cholesterol ; TG: triglyceride; HDL: high-density lipoprotein cholesterol; LDL: low-density lipoprotein cholesterol.

ral arterial distal from bifurcate were also determined. The total number of plaques in carotid and femoral separately in the same patient was counted and their thickness was added up to evaluate the severity of atherosclerosis. The grade of plaques were classified as follows: grade 0 , non or the adding thickness $<1 \mathrm{~mm}$; grade 1 , the adding thickness $1.1-5 \mathrm{~mm}$; grade 2, 5.1-10 $\mathrm{mm}$; grade $3,>10 \mathrm{~mm}$ [3].

\section{Statistics assay}

Data were analyzed with SPSS-13. Values were expressed as mean $\pm \mathrm{SD}$. Comparisons were performed between the groups using Student's t tests, and Chi-Square analysis. Statistical significance was considered when $\mathrm{P}<$ 0.05 .

\section{Results}

\section{Baseline clinical characteristics of the patients}

Patients in study group showed higher prevalence in hypertension history, high systolic blood pressure, diastolic blood pressure, fasting blood glucose, creatinine and urea; with low levels of high-density cholesterol compared with those of controls. There were no differences between sex, 
Table 2. The atherosclerosis of cerebral ischemic patients

\begin{tabular}{llllllll}
\hline Group & $\mathbf{N}$ & $\begin{array}{l}\text { Sum of } \\
\text { carotid } \\
\text { plaques }\end{array}$ & $\begin{array}{l}\text { Left carotid } \\
\text { plaques }\end{array}$ & $\begin{array}{l}\text { Right carotid } \\
\text { plaques }\end{array}$ & $\begin{array}{l}\text { Right carotid } \\
\text { IMT }\end{array}$ & $\begin{array}{l}\text { Sum of } \\
\text { femoral } \\
\text { plauqes }\end{array}$ & $\begin{array}{l}\text { Right } \\
\text { femoral } \\
\text { IMT }\end{array}$ \\
\hline Controls & 100 & $3.40 \pm 4.50^{* * *}$ & $1.59 \pm 2.46^{* * *}$ & $1.81 \pm 2.71^{* *}$ & $0.82 \pm 0.24^{* *}$ & $1.79 \pm 3.64^{*}$ & $0.82 \pm 0.20^{* *}$ \\
Patients & 138 & $6.22 \pm 5.87$ & $3.21 \pm 3.37$ & $3.01 \pm 3.14$ & $0.91 \pm 0.19$ & $3.53 \pm 3.90$ & $0.96 \pm 0.24$ \\
\hline
\end{tabular}

Student' t-test, ${ }^{*} p<0.05,{ }^{* *} p<0.01,{ }^{* * *} p<0.001$

age, BMI, total cholesterol, triglyceride, low-density cholesterol, diabetes history between the two groups (Table 1).

\section{Atherosclerotic plaques and IMT in two groups}

The grade of atherosclerotic plaques and femoral plaques were higher in patients group than in controls $(\mathrm{p}<$ $0.001, p<0.05$, respectively), and a higher mean IMT at both the carotid and femoral sites compared with controls ( $\mathrm{p}$ $<0.01)$. There was significant difference between patients and controls in both left and right carotid plaques ( $\mathrm{p}<0.001$, $\mathrm{p}<0.01$, respectively).

\section{Comparison of the grade of plaques between the two groups}

Compared with that in the control group, the grade of plaques of carotid artery in the ischemic patients was higher $(p<0.01)$. The mean Ridit value of the ischemic patients was $0.577,95 \%$ confidence interval (CI) was $0.6646-0.4894$. The mean value of Ridit in the controls was $0.394,95 \% \mathrm{CI}$ is
0.4969-0.2911. There was significant difference between the two groups. (Table 3, figure 1)

\section{Stable and unstable plaques in two groups}

Carotid atherosclerosis was highly prevalent in study group comparing with controls. The prevalence of plaque of right common artery was $60.14 \%$ in study group, $46 \%$ in the controls $(p<0.05)$. The percentage of plaque of right internal artery was $24.64 \%$ in study group, $11 \%$ in controls $(\mathrm{p}<0.01)$. Unstable plaque of right common carotid and right internal artery was significantly higher in study group than in the controls $(84.34 \%$ vs $60.87 \%, \mathrm{p}<0.01 ; 82.35 \%$ vs $45.45 \%, p<0.05$; respectively). The prevalence of left common carotid in the study group was significantly higher than in the controls $(71.01 \%$ vs $36 \%, p<0.001)$. There was no significant difference of plaque prevalence in left internal carotid between the two groups $(23.19 \%$ vs $13 \%, p>0.05)$. The instability percentage of left common carotid was significantly higher in study group than in controls $(61.59 \%$ vs

Table 3. The grade of the plaques in the patients and controls

\begin{tabular}{lll}
\hline Grade of plaques & Study & Control \\
\hline $0($ or $<1)$ & $21(15.22 \%)$ & $42(42 \%)$ \\
$1(1.1-5)$ & $46(33.33 \%)$ & $34(34 \%)$ \\
$2(5.1-10)$ & $36(26.09 \%)$ & $13(13 \%)$ \\
$3(>10)$ & $35(25.36 \%)$ & $11(11 \%)$ \\
\hline
\end{tabular}

Ridit analysis, 95\% confidence interval $(\mathrm{Cl})$ of the patients with ischemic events: $0.6646-0.4894,95 \% \mathrm{Cl}$ of the controls: $0.4969-0.2911$.

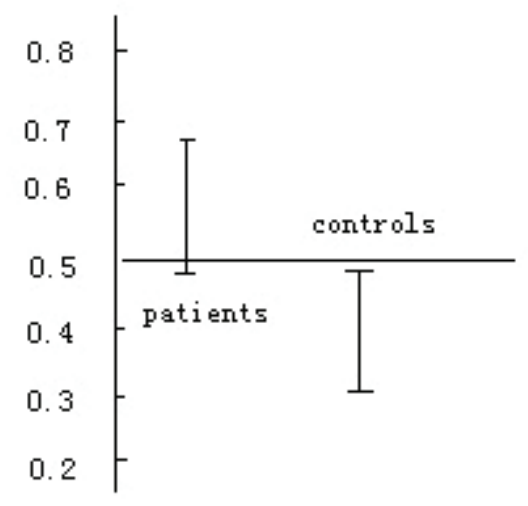

Figure 1. Mean Ridit values in study and control groups, $p<$ 0.01 . 
Table 4. Atherosclerotic plaques of right carotid in acute cerebral ischemic patients

\begin{tabular}{|c|c|c|c|c|c|c|c|}
\hline \multirow{2}{*}{ Group } & \multirow{2}{*}{$\mathbf{N}$} & \multicolumn{3}{|c|}{ Right common carotid (n) } & \multicolumn{3}{|c|}{ Right internal carotid (n) } \\
\hline & & with plaque & soft/mixed & hard & with plaque & soft/mixed & hard \\
\hline Controls & 100 & $46^{*}$ & $16 / 12 * *$ & 18 & $11 * *$ & $3 / 2 *$ & 6 \\
\hline Patients & 138 & 83 & $42 / 28$ & 13 & 34 & $15 / 13$ & 6 \\
\hline
\end{tabular}

Chi-square analysis, ${ }^{*} p<0.05,{ }^{* *} p<0.01$, controls vs patients.

$19 \%, \mathrm{p}<0.001)$. There was no significant difference of stability in left internal carotid between two groups. (Table 4, 5)

\section{Discussion}

Cerebrovascular disease (CVD) or stroke is one of the first three leading causes of death in the past four decades in China. CVD is also the most important cause of disability in the elderly. CVD will still prevail in the future $[4,5]$. The severity of atherosclerosis indicates the risks of the vascular events.

A prompt and accurate diagnosis of carotid artery disease is critical when planning a therapeutic strategy. Even in healthy people, the convenient surveillance of atherosclerosis is important in lifestyle modifications and pharmacological interventions. Physical examination is inaccurate in determining the presence and severity of carotid artery disease. Therefore, reliable imaging tests such as duplex ultrasonography which offer little risk to the patient are required [6]. The meta analysis showed the sensitivity of ultrasonography is approximately $94 \%$ and specificity is approximately $92 \%$ [7]. The Carotid intima-media thickness (CIMT) was found to correlate with transesophageal echocardiography (TEE) markers of aortic atherosclerosis, including complex aortic plaques, and combined cardiovascular sources of embolus (CSE). The optimal CIMT cutoff for detection of CSE on TEE was $0.78 \mathrm{~mm}$ [8]. The plaques are classified as stable and unstable according to the echo feature. Hypoechoic or heterogeneous hypoechoic plaques with an irregular surface or ulcerations have been considered soft/complex plaques at major risk of stroke; homogeneous hyperechoic plaques with smooth surface lesions have been considered simple or hard plaques at minor risk [9]. Study has shown that there is positive correlation between the peripheral artery atherosclerosis and carotid atherosclerosis [10], this implies that monitoring the plaque and thickness of the carotid or the femoral artery is important in preventing the stroke, especially in deciding the prophylactic methods to prevent the re-attack of the stroke.

In this study, the cerebral ischemic patients had higher prevalence in hypertension history, systolic and diastolic blood pressure, blood glucose, creatinine, urea, and had lower level of high-density cholesterol compared with controls. The results indicate the patients are prone to have metabolic disorders. Ishizaka has shown that chronic kidney dysfunction is related to the atherosclerosis [11]. We also showed the prevalence of the atherosclerosis in patients with cere-

Table 5. Atherosclerotic plaques of left carotid in acute cerebral ischemic patients

\begin{tabular}{|c|c|c|c|c|c|c|c|}
\hline \multirow{2}{*}{ Group } & \multirow{2}{*}{$\mathbf{N}$} & \multicolumn{3}{|c|}{ Left common carotid (N) } & \multicolumn{3}{|c|}{ Left internal carotid $(\mathrm{N})$} \\
\hline & & with plaque & soft/mixed & hard & with plaque & soft $/$ mixed & hard \\
\hline Controls & 100 & $36 * * *$ & $8 * * / 11 * * *$ & 17 & 13 & $3 / 5$ & 5 \\
\hline Patients & 138 & 98 & $50 / 35$ & 13 & 32 & $11 / 13$ & 8 \\
\hline
\end{tabular}

Chi-square analysis, ${ }^{* *} p<0.01,{ }^{* * *} p<0.001$, patients versus control. 
bral ischemic disease was higher than in the controls. The unstable plaques that may lead to rupture, such as the soft or complex ones, are more often observed in the stroke patients. The IMT of common carotid or femoral artery were thicker than that in the controls. Our study further verified that atherosclerosis and its characteristic are the important risk factors in stroke onset.

Previous prospective cohort study showed an 11-15\% decrease of stroke risk following every $10 \mathrm{mg} / \mathrm{dl}$ increase of HDL-C [12]. HDL-C protects against atherosclerosis through promoting cholesterol efflux. In addition, the antiinflammatory properties of HDL are important as well [13]. These findings suggest that the severer of the atherosclerosis, the harder in controlling risk factors. For those with vulnerable plaque or severe degree of stenosis ( $\geq 70 \%$ ), we may turn to DSA (digital subtractive angiography) and carotid endarterectomy or stenting.

It is also important to monitor plaque evolution to prevent second stroke or asymptomatic carotid atherosclerosis changing to symptomatic CVD. Furthermore, patients with extracranial carotid atherosclerosis, either symptomatic or asymptomatic, demonstrate altered cerebral perfusion, leading to lacunar infarcts and periventricular and subcortical white matter lesion [14].

\section{Conflict of Interest}

The authors declare no conflicts of interest.

\section{References}

1. Andrei V. Alexandrov. Cerebrovascular ultrsound in stroke prevention and treatment. 2004 by Blackwell Publishing Ltd.

2. Vicenzini E, Ricciardi MC, Puccinelli F, Altieri M, Vanacore N, Di Piero V, Lenzi GL. Common carotid artery intima-media thickness determinants in a population study. J Ultrasound Med 2007;26:427-432; quiz 434.

3. Yi XY, Chen CM, Chi LF, et al. The relationship between carotid atherosclerosis and progressive ischemic stroke. Zhong Hua Shen Jing Ke Za Zhi 2006;39:288391.

4. Jeng JS, Su TC. Epidemiological studies of cerebrovascular diseases and carotid atherosclerosis in Taiwan.
Acta Neurol Taiwan 2007;16:190-202.

5. McClelland S, 3rd. Multimodality management of carotid artery stenosis: reviewing the class-I evidence. J Natl Med Assoc 2007;99:1235-1242.

6. Jaff MR, Goldmakher GV, Lev MH, Romero JM. Imaging of the carotid arteries: the role of duplex ultrasonography, magnetic resonance arteriography, and computerized tomographic arteriography. Vasc Med 2008;13:281-292.

7. Wolff T, Guirguis-Blake J, Miller T, Gillespie M, Harris R. Screening for carotid artery stenosis: an update of the evidence for the U.S. Preventive Services Task Force. Ann Intern Med 2007;147:860-870.

8. Ward RP, Lammertin G, Virnich DE, Polonsky TS, Lang RM. Use of carotid intima-media thickness to identify patients with ischemic stroke and transient ischemic attack with low yield of cardiovascular sources of embolus on transesophageal echocardiography. Stroke 2008;39:2969-2974.

9. Vicenzini E, Ricciardi MC, Puccinelli F, Altieri M, Vanacore N, Di Piero V, Lenzi GL. Sonographic carotid plaque morphologic characteristics and vascular risk factors: results from a population study. J Ultrasound Med 2008;27:1313-1319.

10. Matsumoto M, Inoue K, Moriki A. Associations of brachial-ankle pulse wave velocity and carotid atherosclerotic lesions with silent cerebral lesions. Hypertens Res 2007;30:767-773.

11. Ishizaka N, Ishizaka Y, Toda E, Koike K, Seki G, Nagai R, Yamakado M. Association between chronic kidney disease and carotid intima-media thickening in individuals with hypertension and impaired glucose metabolism. Hypertens Res 2007;30:1035-1041.

12. Amarenco P, Labreuche J, Touboul PJ. High-density lipoprotein-cholesterol and risk of stroke and carotid atherosclerosis: a systematic review. Atherosclerosis 2008;196:489-496.

13. Navab M, Yu R, Gharavi N, Huang W, Ezra N, Lotfizadeh A, Anantharamaiah GM, et al. High-density lipoprotein: antioxidant and anti-inflammatory properties. Curr Atheroscler Rep 2007;9:244-248.

14. Sfyroeras GS, Karkos CD, Gerassimidis TS. Cerebral perfusion patterns in patients with extracranial carotid atherosclerosis and the impact of carotid stenting. A review. J Cardiovasc Surg (Torino) 2008;49:497-502. 